\title{
Amphicrine carcinoma of the stomach and intestine: a clinicopathologic and pan-cancer transcriptome analysis of a distinct entity
}

\author{
Dan Huang $1,2,3$ C Fei Ren ${ }^{1,2,3}$, Shujuan $\mathrm{Ni}^{1,2,3}$, Cong Tan ${ }^{1,2,3}$, Weiwei Weng ${ }^{1,2,3}$, Meng Zhang ${ }^{1,2,3}$, Midie Xu ${ }^{1,2,3}$,
} Lei Wang ${ }^{1,2,3}$, Qinghua $\mathrm{Xu}^{4}$ and Weiqi Sheng ${ }^{1,2,3^{*}}$ (1)

\begin{abstract}
Background and aim: Amphicrine carcinoma, in which endocrine and epithelial cell constituents are present within the same cell, is very rare. This study characterized the clinicopathologic and survival analysis of this tumor, further compared the genetic diversities among amphicrine carcinoma and other tumors.

Materials and methods: The clinicopathologic characteristics and survival outcomes of amphicrine carcinoma in this study were analyzed. The pan-cancer transcriptome assay was utilized to compare the genetic expression profile of this entity with that of conventional adenocarcinoma or neuroendocrine tumors.

Results: Ten cases (all in male patients) were identified in the stomach or intestine, with a median patient age of 62 years. There were characteristic patterns in the tumors: tubular, fusion or single-file growth of goblet- or signet ring-like cells. Four tumors were classified as low-grade and 6 as high-grade according to the histologic architecture. All cases were positive for neuroendocrine markers (synaptophysin and chromogranin A) and showed intracellular mucin in the amphicrine components. Four cases exhibited mRNA expression patterns showing transcriptional homogeneity with conventional adenocarcinomas and genetic diversity from neuroendocrine tumors. During the follow-up period, 3 patients died of disease, all of whom had high-grade tumors. Patients with high-grade amphicrine carcinoma had worse outcomes than those with low-grade tumors.

Conclusions: This study confirms the morphological, immunostaining and transcriptome alterations in amphicrine carcinoma distinct from those in conventional adenocarcinomas and neuroendocrine tumors, but additional studies are warranted to determine the biological behavior and therapeutic response.
\end{abstract}

Keywords: Amphicrine, Gastrointestinal tract, Prognosis, Pan-cancer transcriptome analysis

\section{Background}

Although rare, mixed exocrine-neuroendocrine tumors have been previously described in the gastrointestinal tract [1]. Almost 30 years ago, Lewin proposed a nomenclature for dividing this unique type of tumor into three groups [2]: mixed or composite tumors, collision tumors

\footnotetext{
*Correspondence: Shengweiqi2006@163.com

${ }^{1}$ Department of Pathology, Fudan University Shanghai Cancer Center,

270 Dong An Road, Shanghai 200032, People's Republic of China

Full list of author information is available at the end of the article
}

and amphicrine tumors. Amphicrine neoplasms have been described as tumors with exocrine and neuroendocrine components in the same cell. This pattern contrasts with that in composite tumors or collision tumors, in which 2 different cellular components are admixed or juxtaposed. Since only a few studies have included amphicrine neoplasms, the use of the term "amphicrine tumor" in some studies and "amphicrine carcinoma" in others leads to great confusion in interpreting and understanding this neoplasm [3-5]. We propose "amphicrine 
carcinoma" as the designation to highlight its aggressive behavior.

With both neuroendocrine and exocrine differentiation, amphicrine carcinomas are significantly more likely to have unique features in histopathology. However, the descriptions of amphicrine carcinomas in terms of morphology and immunophenotype have, to date, been limited in previous reports. In the clinic, the dual nature of these tumors is still largely unrecognized, and there is no unified concept of how to treat patients with amphicrine carcinoma.

To gain a better understanding of the biological properties of amphicrine carcinoma, it is essential to study the genetic profiles of these tumors. Recently, close genetic relations have been revealed in mixed adenoneuroendocrine carcinomas (MANECs) and adenocarcinomas [6]. Another next-generation sequencing study focusing on somatic mutations and driver genes also suggested a monoclonal origin for different components of MANEC [7]. However, a comparison of molecular characteristics, especially mRNA levels, between amphicrine carcinomas and adenocarcinomas or neuroendocrine tumors is needed.

Our study, the largest case series to date, aimed to explore the clinicopathological features of amphicrine carcinoma in the stomach and intestine via hierarchical clustering analysis using a pan-cancer transcriptome panel in an effort to more appropriately define the specific morphology, clinical behavior and genetic differences of this neoplasm from other neoplasms.

\section{Materials and methods Case selection}

This study was performed in accordance with local ethical and legal requirements after approval by the Ethics Committee of Fudan University Shanghai Cancer Center (FUSCC), and written informed consent was obtained from all participants or their appropriate surrogates. A total of ten cases of amphicrine carcinoma of the stomach or intestine were retrieved from the consultation and surgical pathology files of the Department of Pathology at FUSCC between 2009 and 2017. Available medical records, including imaging study reports, were reviewed to obtain clinical data such as age, sex, presenting symptoms, endoscopic descriptions, treatment, and outcome; for the consultation cases, contributing physicians were contacted.

\section{Pathologic features}

Available gross images, descriptions and histologic sections were reviewed by three pathologists (authors: DH, FR and SJN) to confirm the diagnosis and further characterize the histological findings. The pathologic criteria for diagnosis of amphicrine carcinoma requires exocrine and neuroendocrine features in the same neoplastic cell, which shows a divergent immunophenotype [1]. According to histologic features, our cases were divided into low, intermediate and high grades using the grading system recommended by Yozu [8]. This methodology is used to grade appendiceal goblet cell carcinomas by assessing the proportion of the tumor with tubular or clustered growth. Tumors with $>75 \%$ tubular or clustered growth were classified as having a low-grade pattern, which is characterized by small and cohesive clusters of goblet cells with or without lumina. The cells in these clusters had low to at most moderate cytologic atypia and infrequent mitoses, sometimes with peripheral localization of nuclei. Tumors with $50 \%$ to $75 \%$ tubular growth were classified as having an intermediate-grade pattern, and tumors with $<50 \%$ tubular growth were classified as having a high-grade pattern. These growth patterns deviated from the low-grade pattern and showed several forms, including single-file or sheet-like growth of signet ringlike cells. Clusters of tumor cells, especially in mucinpoor areas, had increased cytologic atypia and increased mitotic activity.

\section{Immunohistochemistry}

Immunohistochemistry for pankeratin (clone AE1/3, 1:150, Dako), CgA (clone LK2H101+PHE5, Roche), Syn (clone MRQ-40, 1:400, Roche), CD56 (clone 123C3, 1:80, Dako) and Ki67 (clone 30-9, Roche) were performed using a Ventana BenchMark XT Automated Staining System. Alcian blue staining was performed to evaluate mucin content using an Alcian blue staining kit (BASO) following the manufacturer's instructions.

\section{Pan-cancer transcriptome assay}

The genetic data generated for amphicrine carcinomas were compared with data from a set of neuroendocrine tumors and gastric adenocarcinomas, which were genetically analyzed by the same 90 -gene real-time PCR assay [9]. In brief, manual macrodissection of tumor-rich areas from unstained slides of formalin-fixed, paraffin-embedded tissue was performed under microscopy with guidance from hematoxylin and eosin staining. Total RNA was isolated from formalin-fixed, paraffin-embedded (FFPE) tissue sections using an FFPE Total RNA Isolation Kit (Canhelp Genomics, Hangzhou, China). The concentration and purity of total RNA were determined by spectrometry according to the manufacturer's instructions. Next, cDNA was generated from isolated total RNA using a high-capacity cDNA Reverse Transcription Kit with RNase Inhibitor (Applied Biosystems, Foster City, CA, United States). The A260/A280 of total RNA isolated from tissue sections from amphicrine carcinoma, NET 
and gastric cancer patients was 1.89-2.00. The expression level of each of the 90 genes was measured in an Applied Biosystems 7500 Real-Time PCR system using TaqMan Gene Expression Assays (Applied Biosystems). Normalized gene expression intensities were shifted to set the mean to 0 and rescaled to set the STD to 1 to enhance the expression differences. The average linkage hierarchical clustering method was performed, where the metric of similarity was the Pearson correlation between every pair of samples. In addition, relative mRNA expression intensities were triple detected for each specimen of all samples, including amphicrine carcinoma (AC), neuroendocrine tumor (NET) and stomach adenocarcinoma (STAD) samples.

Biological network analysis and KEGG (Kyoto Encyclopedia of Genes and Genomes) pathway analysis were performed using NetworkAnalyst software (version 3.0) (Cite https://doi.org/10.1093/nar/gkz240). Protein-protein interactions have been retrieved from IMEx Interactome Database (Cite https://doi.org/10.1093/nar/gks11 47). A minimum network was generated by keeping seed proteins as well as minimum essential non-seed proteins to study the fundamental interactions.

\section{Statistical analysis}

Statistical analyses to compare clinicopathologic characteristics and overall survival were performed using SPSS (version 20.0, IBM). Means and ranges are used to describe quantitative variables. Overall survival curves were generated by the Kaplan-Meier method, and the log-rank test was used in difference analyses. A $P$ value of $<0.05$ was considered statistically significant.

\section{Results}

\section{Clinical features}

The clinical features and staging parameters for our ten amphicrine cancer cases are summarized in Table 1. All patients were male, with a mean age of 63 years (ranging from 56 to 69). The presenting symptoms in seven patients included upper abdominal pain, hematochezia and hematemesis. The eight gastric neoplasms were located throughout the stomach, and the antrum was the most commonly involved region. Another two cases in the intestine arose from the rectum. According to the histologic evaluation of tumor grades, most low-grade tumors were in an early $\mathrm{T}$ stage ( $\mathrm{T} 1$ or $\mathrm{T} 2)$. No patient had nodal or distant metastasis at presentation; however, one patient with stage IIIA disease had one lymph node involved. Lymphovascular invasion was present in only that patient (25\%), and perineural invasion was not observed in any low-grade sample. In contrast, the highgrade group and mixed group had a high proportion of late-stage disease. The percentage of patients with lymph node involvement was increased (75\%), as did the percentage of patients with synchronous distant metastasis (33\%). Perineural invasion was observed in 2 high-grade tumors, and lymphovascular invasion was present in 3 cases.

\section{Pathologic findings}

Grossly, the tumor sizes ranged from 2 to $5 \mathrm{~cm}$ (mean, $3.6 \mathrm{~cm}$ ) in the maximum dimension. In the 9 cases for which endoscopic or gross information was available, the ulcerative nature of the tumor was described in seven (Fig. 1a). The remaining two tumors were documented as fungating lesions. After assessment of tubular and clustered components, 4 tumors were categorized as lowgrade, 6 as high-grade, and none as intermediate-grade.

The histology was somewhat complicated but showed three components consisting of three types of neoplasms: (1) low-grade amphicrine carcinoma (may mix with other components but less than $30 \%$ of tumor cell population); (2) high-grade amphicrine carcinoma (may mix with other components but less than 30\% of tumor cell population); and (3) mixed amphicrineneuroendocrine carcinoma (amphicrine carcinoma and other carcinoma, each of which according for more than $30 \%$ of tumor cell population). After assessment of the components and grades, 4 tumors were in the low-grade group, 4 were in the high-grade group, and 2 were in the mixed group (Table 2). In the lowgrade group, one patient had another minor conventional adenocarcinoma component comprising $5 \%$ of the tumor. By definition, the low-grade group included tumors with up to $25 \%$ high-grade components, but none of the included tumors showed combination with any high-grade components. The most common histologic architectures in the low-grade category were tubular growth with intracellular mucin and peripheral placement of nuclei (Fig. 2), which resembled goblet cell carcinoid/carcinoma in the appendix. None of the cases in this group had single-file infiltration by signet ring-like cells. In 2 cases with an extracellular mucin pool, the tumor clusters maintained their cohesive, uniform appearance, and resembled disrupted intestinal crypts. In the high-grade group, one case presented one area of conventional adenocarcinoma (5\%) and another area of low-grade amphicrine cancer characterized by tubular structures lined by goblet-like cells (45\%). Another high-grade case had a low-grade component of 5\%. Some nontubular growths were common among the high-grade tumors, which deviated from the architecture in the low-grade group. The most frequent forms were fusion of goblet cell clusters and disorganized growth by signet ring-like cells. Single files of goblet cells or signet ring-like cells were 


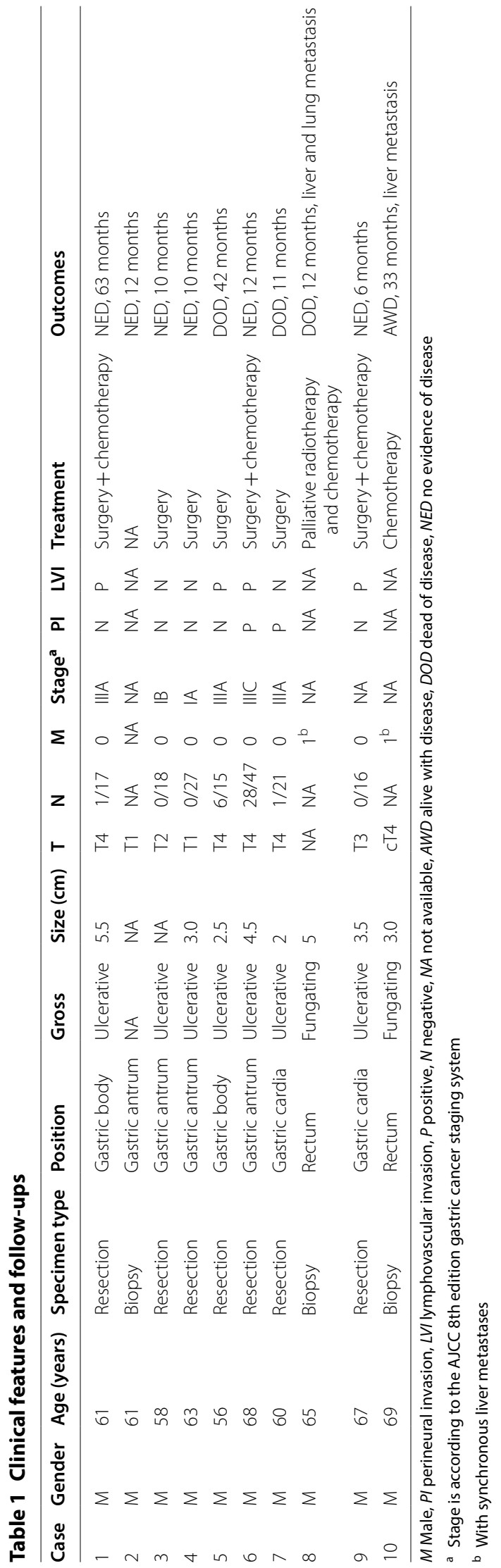


a

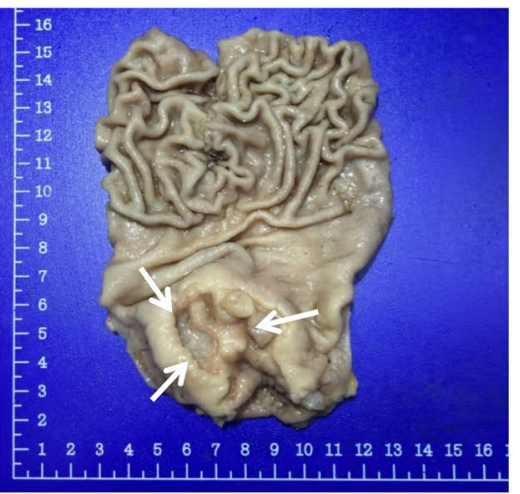

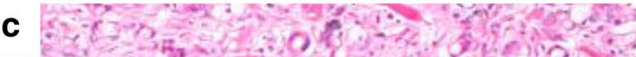

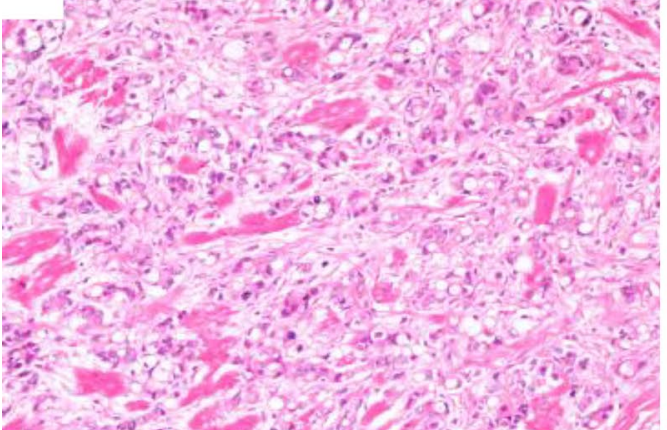

b

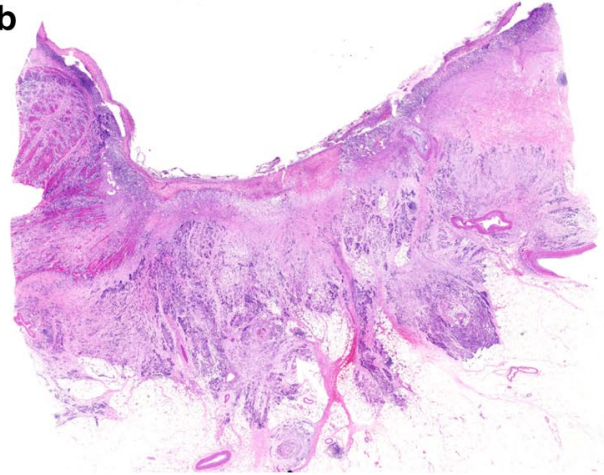

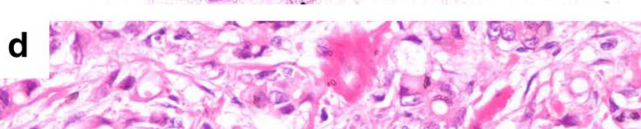

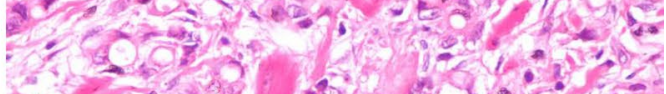

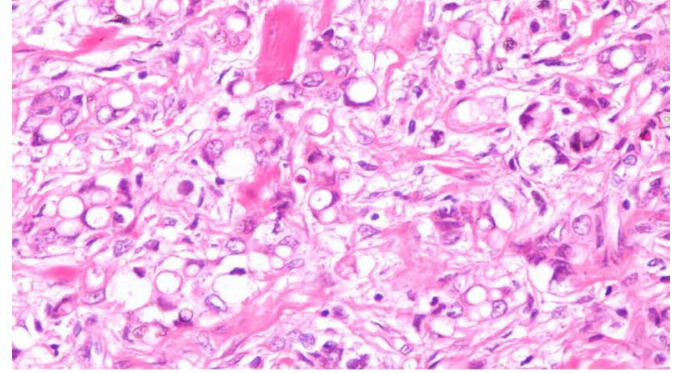

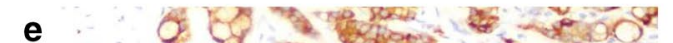

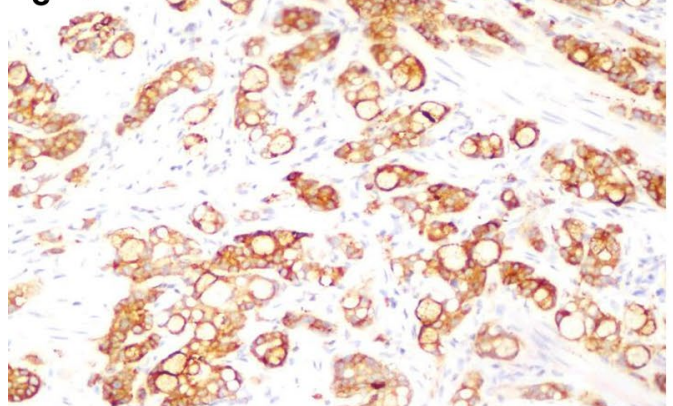

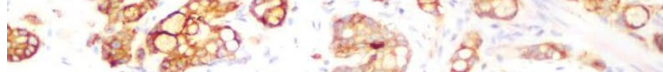

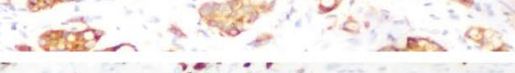
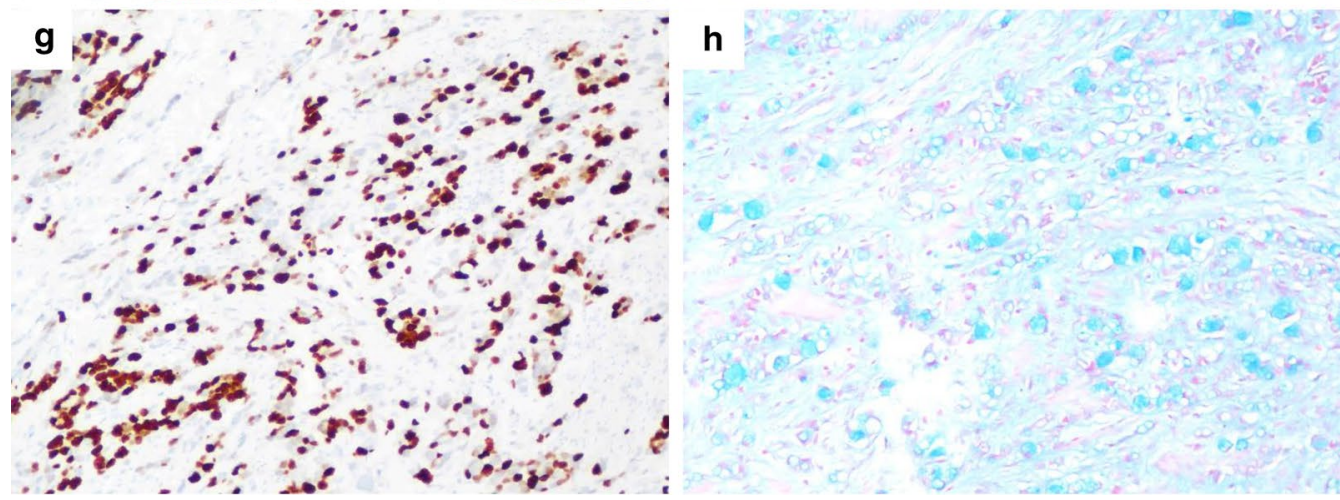

Fig. 1 Amphicrine carcinoma with a high-grade pattern (case 6). a Ulcerative mass in the gastric angle, gross appearance. b Destructive infiltration with extension into the subserosal tissue. $\mathbf{c}$ Fusion and disorganized growth of amphicrine carcinoma cells, $\times 400$. $\mathbf{d}$ Infiltrating signet ring-like cells with nuclei compressed to the periphery by abundant intracellular mucin, $\times 400$. e Positive staining of synaptophysin, $\times 200 . \mathbf{f}$ Focal positive staining of chromogranin A, $\times 200 . \mathbf{g}$ Immunostaining of Ki67, $\times 200$. h Staining of intracellular and extracellular mucin by Alcian blue, $\times 200$ 


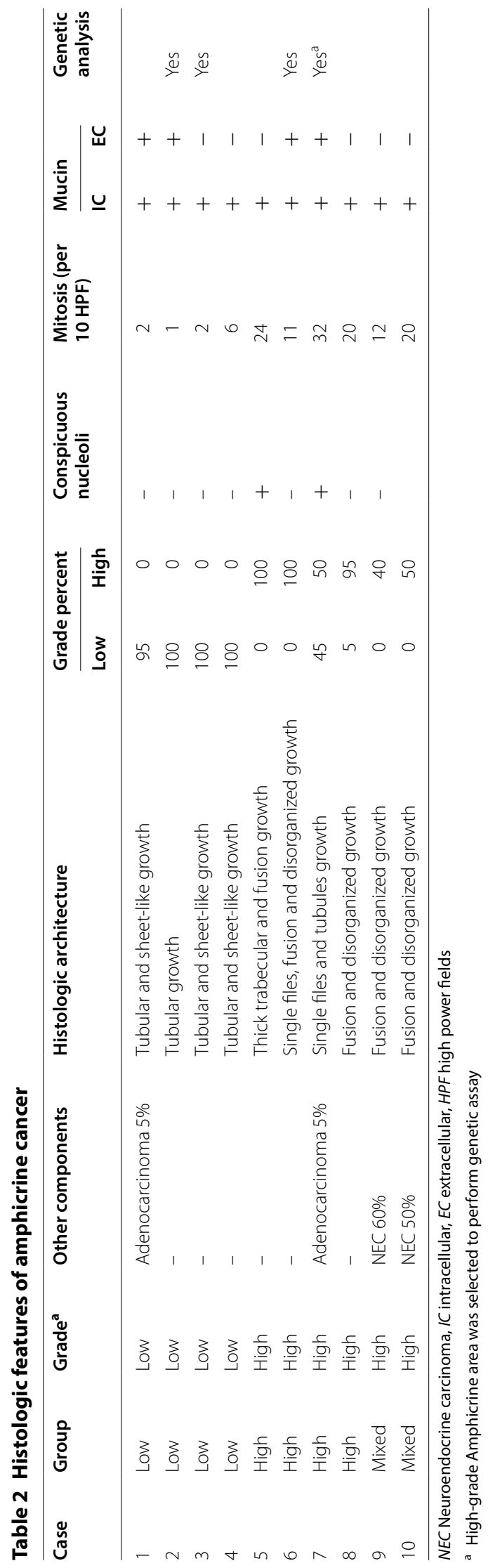




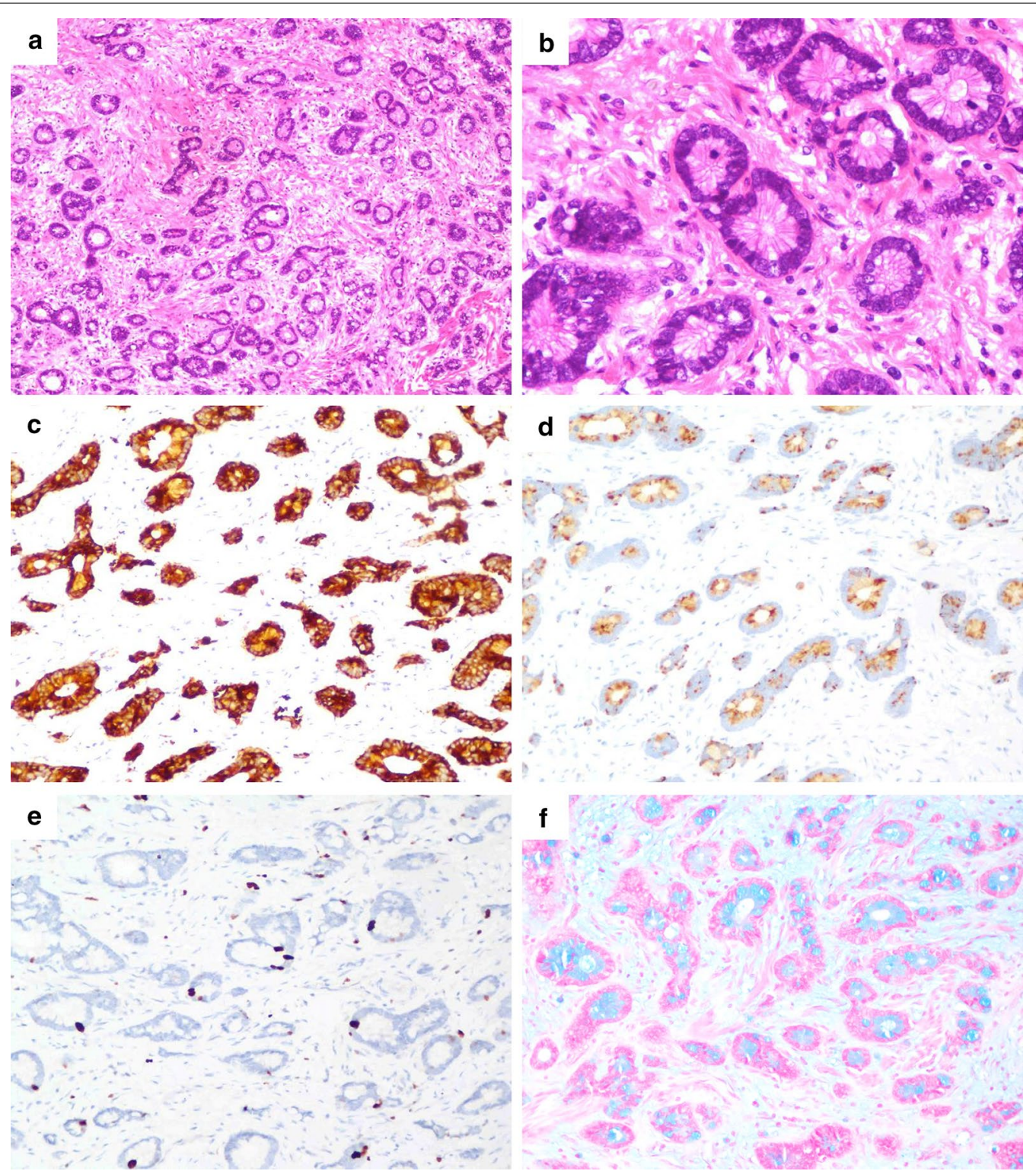

Fig. 2 Amphicrine carcinoma with a low-grade pattern (case 4). a Small tumor clusters with lumens and peripheral placement of nuclei, $\times 100$. b Well-formed tubules comprising goblet-like mucinous cells, $\times 400$. c Diffuse positive staining for synaptophysin, $\times 200$. $\mathbf{d}$ Paranuclear dot-like immunostaining of chromogranin A, ×200. e Immunostaining of Ki67, ×200. f Intracellular positivity for Alcian blue staining, $\times 200$

also fairly common (Fig. 1). Less common patterns included mucin-poor areas composed of tumor nests with high cytologic grade that resembled conventional adenocarcinoma and tumor clusters floating in mucin formed by signet ring-like cells with nuclear atypia and mitotic frequency. There were 2 cases of mixed amphicrine-neuroendocrine carcinoma that had a high-grade amphicrine component intermixed with areas of conventional neuroendocrine carcinoma (NEC), comprising $50 \%$ and $60 \%$ of the tumor (Fig. 3). Similar to the growth patterns in the high-grade group, the amphicrine components were aggregates and fusions of goblet cells forming complex disordered structures, which differentiated these areas from typical NEC areas in histologic architecture.

The immunohistochemical staining results for all cases is summarized in Table 3. All tumor components were positive for cytokeratin (AE1/3). Amphicrine components in all cases had at least focal or patchy positivity for synaptophysin and chromogranin A. CD56 staining, if performed, also showed cytoplasmic positivity in most tumors. Those neuroendocrine markers 


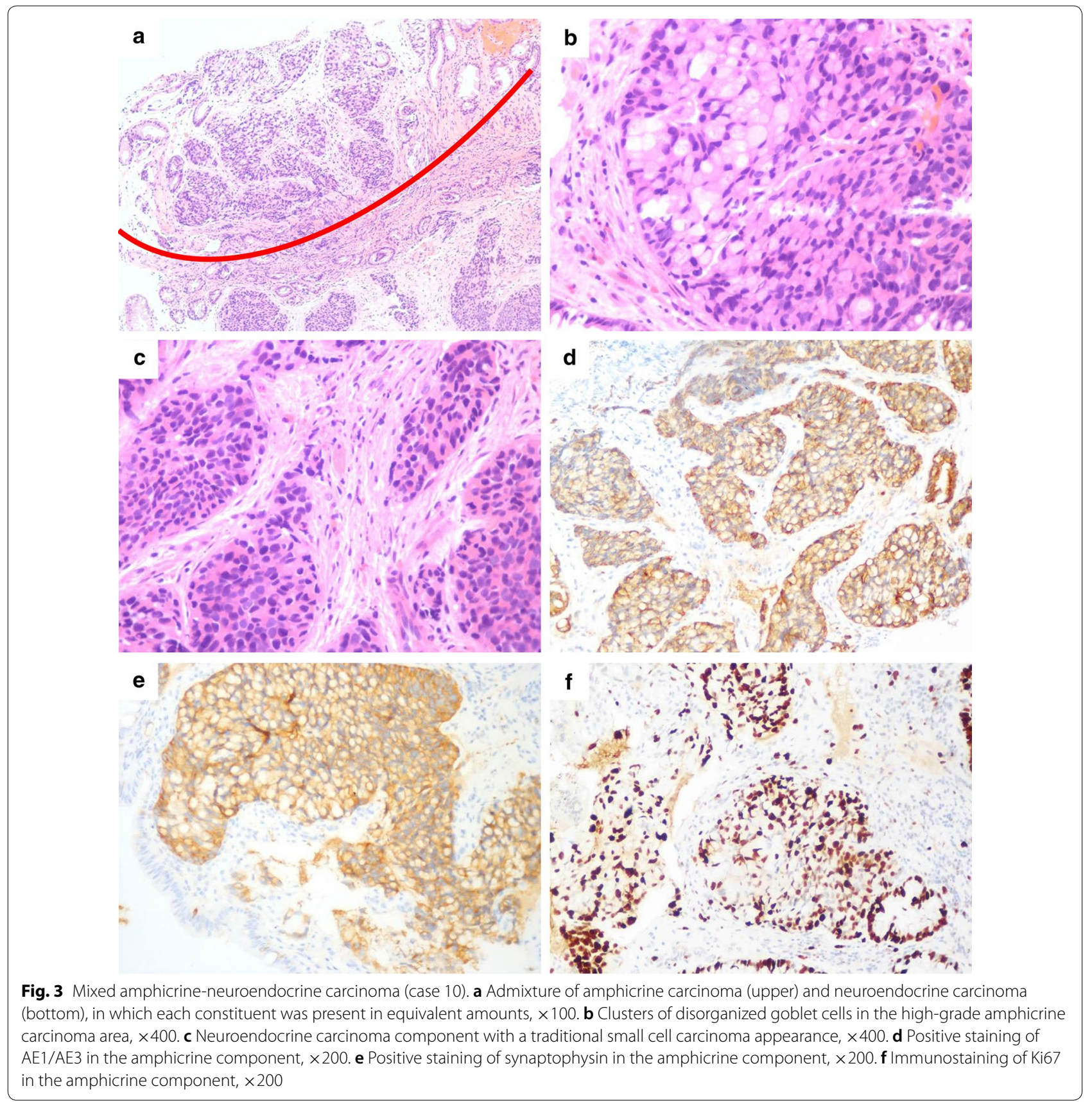

did not differ between the low-grade and high-grade groups, only indicating the amphicrine differentiation of tumor cells. Mucin was visualized by Alcian blue staining, identifying the goblet cells and signet ringlike cells with true intracellular mucin in amphicrine components. The range of the Ki67 index was $5-40 \%$ in low-grade tumors and $20-70 \%$ in high-grade tumors, suggesting a difference in cell proliferation between the two groups.

\section{Pan-cancer transcriptome analyses}

A previous study established a 90-gene expression signature to accurately classify a broad spectrum of tumor types [10]. In our research, the genetic data generated for amphicrine carcinomas, 4 cases with available blocks (Table 2), were compared with data from a set of four neuroendocrine tumors and four gastric adenocarcinomas. The average linkage hierarchical clustering method was performed, where the metric of similarity was the 
Table 3 Immunohistochemical and special staining results

\begin{tabular}{|c|c|c|c|c|c|c|}
\hline Case & $\mathrm{AE} 1 / 3$ & $\operatorname{Cg} A^{a}$ & Syn ${ }^{a}$ & $\mathrm{CD} 56^{\mathrm{a}}$ & $A B^{a}$ & $\mathrm{Ki}-67^{\mathrm{a}}$ \\
\hline 1 & + & + & + & - & + & $5 \%$ \\
\hline 2 & + & + & + & / & + & / \\
\hline 3 & + & + & + & - & + & $35 \%$ \\
\hline 4 & + & + & + & + & $+(\mathrm{P})$ & $40 \%$ \\
\hline 5 & + & + & + & / & $+(\mathrm{P})$ & $20 \%$ \\
\hline 6 & + & $+(\mathrm{P})$ & + & / & + & $70 \%$ \\
\hline 7 & + & + & + & $+(\mathrm{F})$ & + & $30 \%$ \\
\hline 8 & + & + & + & / & $+(\mathrm{P})$ & $60 \%$ \\
\hline 9 & + & $+(\mathrm{P})$ & + & $+(\mathrm{P})$ & $+(\mathrm{P})$ & $60 \%$ \\
\hline 10 & + & $+(\mathrm{F})$ & + & + & $+(\mathrm{P})$ & $70 \%$ \\
\hline
\end{tabular}

$A B$ Alcian blue, CgA chromogranin A, $F$ focal ( $<10 \%$ labeling), $P$ patchy ( 11 to $49 \%$ labeling), Syn synaptophysin

a In amphicrine component; / indicates not performed

Pearson correlation between the 90-gene expression profiles of the samples (Fig. 4a). The heatmap representation of mRNA expression shows that all amphicrine carcinomas and conventional adenocarcinomas were clustered together. Relatedness of clusters was identified between amphicrine carcinomas and NETs. The relative expression intensity of the 12 genes with the greatest variation was used to differentiate related groups in these samples (Fig. 4b). The expression levels of the NPTX2, PCP4, ISL1, IGFBP2, GPX3, VEGFA, ID4 and GPM6B genes were synchronously decreased in the AC and STAD groups compared with their expression levels in NET entities. High levels of CEACAM5, AGR2, CXCL14 and S100P gene expression were simultaneously observed in the AC and STAD groups, while the NET group had significantly lower expression levels of those genes. In the further analysis of the minimum protein-protein network, 12 genes were generated as the seeds, and 10 of them are centered on VEGFA node (Fig. 4c). Next, enrichment analysis of genes was performed in the network. It revealed that the most significant KEGG pathways were signaling pathways regulating pluripotency of stem cells, TGF- $\beta$ signaling pathway, pathways in cancer, etc. (Additional file 1: Table S1). Upon inspection, it was evident that amphicrine carcinoma exhibited transcriptional homogeneity with conventional adenocarcinoma and genetic diversity from NET. Moreover, the gene profiles in the low-grade and high-grade groups were indistinguishable in our limited cohort, exhibiting similar mRNA expression levels in low-grade (cases 2 and 3) and high-grade (cases 6 and 7) tumors.

\section{Treatment and survival outcomes}

Regarding treatment, seven patients underwent complete resection, and three of these received chemotherapy after surgery. Two patients received adjuvant chemotherapy or chemoradiotherapy after biopsy. One patient with early-stage disease did not receive any treatment. Survival information was available for all patients (Table 1 ), with a follow-up period ranging from 6 to 63 months. A total of 3 patients died of disease-at 11, 12 and 42 months, and all had tumors with a high-grade pattern. Seven patients were alive after a follow-up period ranging from 6 to 63 months. Of these patients, 6 had experienced no recurrence or metastasis after treatment; 4 of these patients had low-grade tumors. The survival rate was $100 \%$ in the low-grade group, compared to $50 \%$ in the high-grade group $(\mathrm{P}<0.05)$. Kaplan-Meier survival curves showed a dramatic difference in overall survival between the low-grade and high-grade groups (Fig. 5), suggesting that high-grade morphology is associated with poorer prognosis.

\section{Discussion}

The coexistence of endocrine and exocrine secretory products within single cells was first suggested by Feyrter in 1938 [11]. Later, Ratzenhofer advocated the term "amphicrine" for cells synchronously displaying exocrine and endocrine differentiation [12]. In 1987, Lewin proposed a simple nomenclature for dividing mixed exocrine-neuroendocrine tumors into three groups [2]: mixed or composite tumors, collision tumors and amphicrine tumors. Then, Lewin and Appelman revised the nomenclature into five categories [13], including (1) carcinomas with interspersed NE cells, (2) composite glandular-endocrine carcinomas, (3) collision tumors, (4) amphicrine tumors, and (5) combinations of the first 4 . Since that time, investigators have further subdivided this unique tumor into additional categories $[1,14]$. However, the terminology and classification of amphicrine tumors are still controversial. The terms that have been used to describe amphicrine neoplasms in the GI tract include 


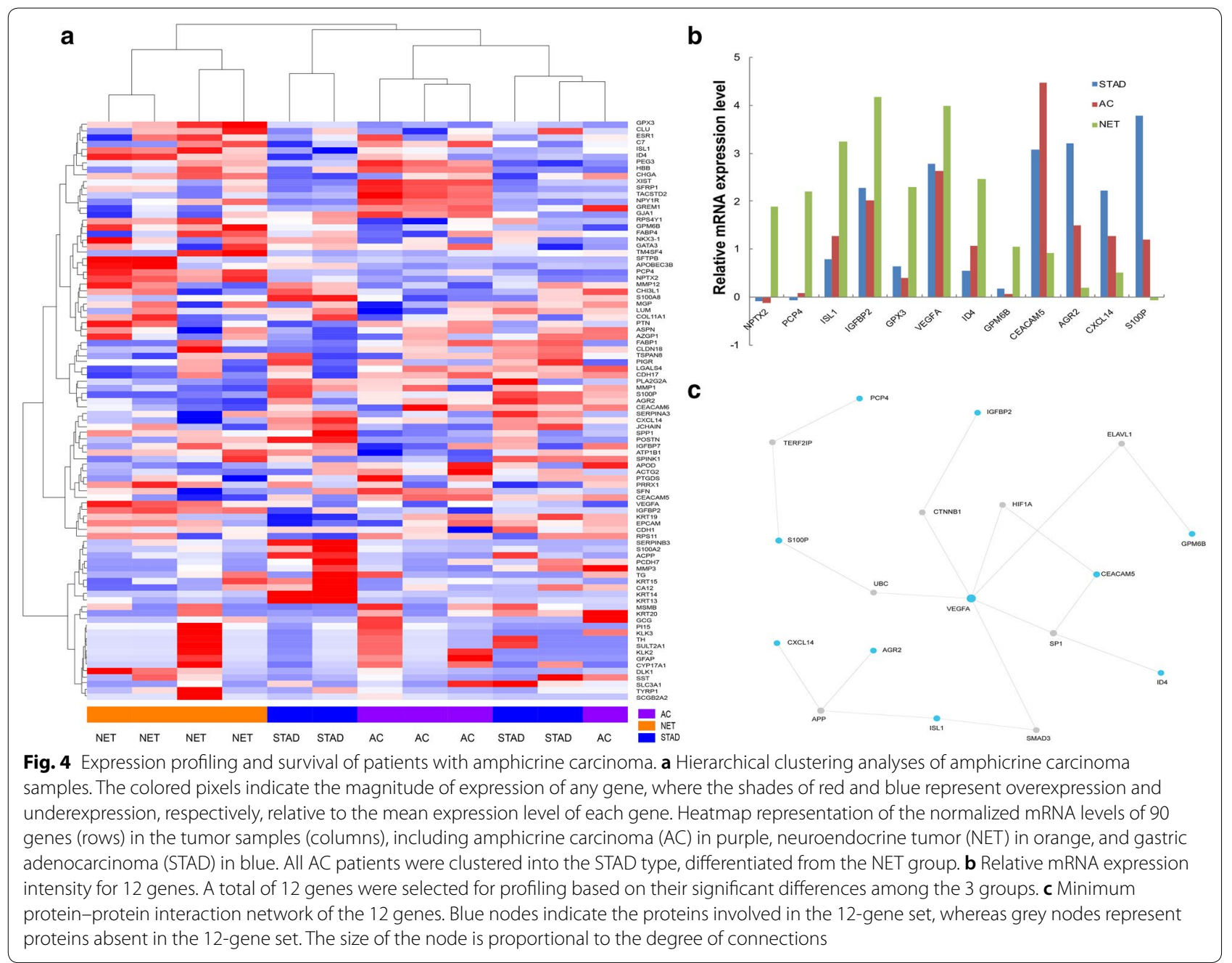

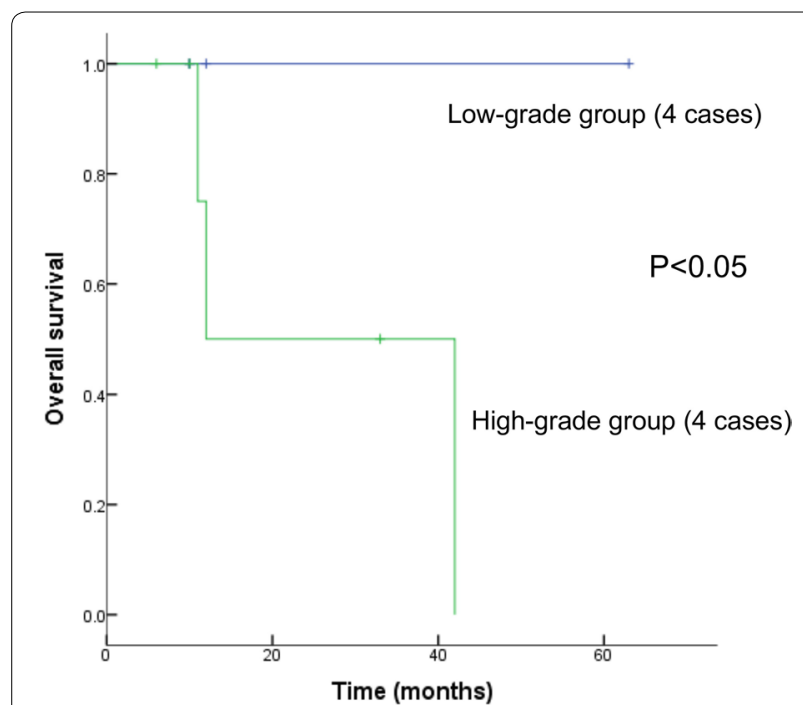

Fig. 5 Kaplan-Meier survival curves for the cohort based on tumor grade, comparing the low-grade group with the high-grade group goblet cell carcinoid (GCC) [15], goblet cell carcinoma [16], amphicrine tumor $[3,4]$ and amphicrine carcinoma $[5,17]$. The current (2010) World Health Organization designated only appendiceal amphicrine neoplasms as GCCs, classified as a special subtype both in neuroendocrine neoplasms (NENs) and in adenocarcinomas [18]. Further studies revealed the aggressive clinical behavior of GCCs $[15,19]$, which supported the change from "goblet cell carcinoid" to "goblet cell carcinoma" $[8,16]$. This terminology facilitates the staging and clinical treatment of these tumors as adenocarcinomas, not as NENs, with the AJCC recommendations [20]. For non-appendiceal GI tract tumors, different terms produce additional confusion, even potentially misleading diagnosis and treatment. Some investigators used the term "extraappendiceal GCC", which should, in the words used, carefully differentiate these tumors from extra-appendiceal metastasis of primary appendiceal GCC [21]. Since the word "amphicrine" provides an appropriate description of hybrid epithelial-neuroendocrine neoplasms, we 
advocate using the term "amphicrine carcinoma", a term that accounts for these unique lesions of intermediate malignancy, for these cases.

In this study, we analyzed 10 cases, including 8 lesions arising in the stomach and 2 in the intestine. All of our patients were male, with a median age of 62 years (range 56-68 years). The predominant locations were the antrum in the stomach and the rectum in the intestine. Most patients presented with advanced-stage disease and lymph node metastasis. Similarly, involvement of lymphoid tissue was found in the previous case reports [4, 22]. Morphologically, these cases could be divided into pure amphicrine carcinoma and amphicrine carcinoma mixed with neuroendocrine carcinoma (NEC) or adenocarcinoma. The latter mixed pattern has also been observed in the appendix (adenocarcinoma ex-GCC), which is associated with a worse outcome than pure GCC $[9,23]$. In terms of morphologic criteria, amphicrine carcinomas with bidifferentiation indicated by staining were difficult to classify with the current grading system based on common adenocarcinoma. For appendiceal GCC tumors, several grading systems have been proposed to classify patients into prognostically relevant groups [24, 25]. We followed Yozu's grading system [8], assessing the proportion of the tumor exhibiting tubular or clustered growth, to subgroup our cases into 6 high-grade cases and 4 low-grade cases. To date, limited survival information has been available in studies of amphicrine carcinomas of the stomach and intestine. In a literature review, Nugent et al. [26] suggested that these carcinomas behave less aggressively, with a better outcome, than other tumors in these locations. In contrast, previous studies in appendiceal GCC indicated that tumor grade is the major influence on clinical presentation and prognosis $[8,27]$. Our results supported the latter view that the histologic grade is closely correlated with overall survival. As shown in case 3 , this patient had a pure low-grade lesion in stage IIIA (T4N1), but lived for 63 months. Thus, histologic grading might be exerted an effect on tumor survival in amphicrine carcinomas.

The morphologic features of the amphicrine component resemble the previously described clinicopathologic findings in case reports of stomach neoplasms. Young et al. [28] reported a case of amphicrine carcinoma of the stomach that was arranged in a classic carcinoid pattern of solid nests and tubules and confirmed to exhibit biphasic differentiation by electron microscopy. Fujiyoshi et al. [29] also reported two composite carcinomas of the stomach with a GCC component formed by goblet carcinoid cells in tubules and rosette-like structures. In our cases, the histological characteristics distinguished different grades. All low-grade amphicrine cancers had the morphologic appearance of tubular growth. Some high-grade cases showed single-file cell infiltration, which represents a pattern of cancer cell spread. In addition to their distinct architectural patterns, low-grade tumors were more likely to show a lower cytologic grade, lower N/C ratio and less mitosis (average of 2.75/10 HPF) than high-grade tumors with more malignant aspects. However, there was no difference in the presence of intracellular or extracellular mucin. All cases exhibited expression of at least one neuroendocrine marker (Syn, CgA or CD56), and no differences were found in the levels of these markers among different grades. Alcian blue staining, which was available in all examined cases, was helpful in identifying exocrine function. Notably, an increase in the mitosis rate and Ki67 proliferation index was readily observed in high-grade cases, with an average index of $22 \%$ in the low-grade group and $52 \%$ in the high-grade group. However, the role of the Ki67 index was quite different in studies of appendiceal GCC; thus, the prognostic value of the proliferation rate is still controversial [24, 30]. Although in limited cases in our study, Ki67 index seems not a predictive marker for prognosis in amphicrine carcinomas, which needs further studies.

The frequency of amphicrine carcinoma of the stomach and intestine may be underestimated in current diagnostic practice. Indeed, the amphicrine component may be misinterpreted as a signet ring cell formation of an adenocarcinoma if expression of neuroendocrine markers is not found by immunohistochemistry. Notably, a relatively high frequency of neuroendocrine positivity was found in previous studies of signet ring cell carcinomas; these studies reported immunostaining for neuroendocrine markers in approximately $40 \%$ of cases [31]. One study limited to staining for chromogranin A, a sensitive marker of neuroendocrine differentiation, also demonstrated focal or diffuse immunopositivity in $37.3 \%$ of gastric signet ring cell carcinomas, including $6 \%$ with staining in more than half of neoplastic cells [32]. In these previously cited studies, cells with neuroendocrine staining in signet ring carcinoma appear to represent amphicrine differentiation, demonstrating the distinct cytologic and architectural features of these tumors from those of composite tumors of mixed signet ring cell carcinoma and NEC. In our study, amphicrine carcinomas with other adenocarcinoma or NEC components were found in 4 of 10 cases, showing a high frequency of mixed growth patterns. Thus, pure amphicrine carcinoma is unusual, but the amphicrine component in mixed form is not as rare due to underdiagnosis and neglect in reporting.

Amphicrine carcinoma is a unique entity with distinct biological and histological features. However, its genetic background and molecular relationship to adenocarcinoma/NEC is largely unknown. Previous studies 
revealed that different components of mixed adenoneuroendocrine carcinomas have similar mutation profiles, suggesting a developmental relationship between neuroendocrine carcinoma and conventional adenocarcinoma $[6,7,33]$. Recently, a study of appendiceal goblet cell carcinoids revealed the mutational distinction between goblet cell carcinoids and neuroendocrine neoplasms/adenocarcinomas [34]. These investigations appear to be quite limited and controversial. To comprehensively analyze the transcriptomic profile of amphicrine carcinoma, we performed pan-cancer transcriptome analyses in 12 patients, including patients with amphicrine cancer, adenocarcinoma and NEC. This gene expression signature was established in a comprehensive database integrating microarray- and sequencing-based gene expression profiles. Previous studies had demonstrated the excellent performance of the 90-gene expression signature for identification of tumor origin [10, 35]. After hierarchical clustering of the gene expression magnitudes, the pan-cancer panel reflected the similarity between the mRNA expression profile in amphicrine carcinoma and traditional adenocarcinoma, with no relationship between the amphicrine carcinoma and NET profiles. In the minimum protein-protein network and enrichment analysis, genes from amphicrine carcinoma were mostly related to VEGFA node and pathways in cancer. These findings provide additional insight into the nature of amphicrine carcinoma. Interestingly, the possibility that amphicrine carcinomas are genetically related to adenocarcinomas raises the question of whether adenocarcinoma-targeted treatments would display a response in amphicrine entities with molecular alterations.

\section{Conclusion}

In summary, amphicrine carcinoma is a distinct clinicopathologic entity in the stomach and intestine. The survival outcome of this malignancy is related to the histologic grade, and its rarity might result in significant underdiagnosis, especially in mixed form. The pan-cancer transcriptome analysis revealed that amphicrine carcinoma is genetically linked to adenocarcinoma instead of to neuroendocrine tumors. Further studies are warranted to determine whether these tumors may be susceptible to adenocarcinoma-targeted treatments.

\section{Supplementary information}

Supplementary information accompanies this paper at https://doi. org/10.1186/s12935-019-1031-7.

Additional file 1: Table S1. Enriched KEGG pathways of 18 genes within network ( $p$ value $<0.05$ )
Abbreviations

MANEC: mixed adenoneuroendocrine carcinomas; AC: amphicrine carcinoma; NET: neuroendocrine tumor; STAD: stomach adenocarcinoma; GCC: goblet cell carcinoid; NEN: neuroendocrine neoplasm; NEC: neuroendocrine carcinoma.

\section{Acknowledgements}

We thank Xu Cai and Xueke Zhou for technical assistance in performing the staining.

\section{Authors' contributions}

Conception and design: WQS, DH. Development of the methodology: FR, SJN CT, WWW, MZ, MDX, QHX. Analysis and interpretation: DH, QHX. Writing and review: $\mathrm{DH}$. Study supervision: WQS. All authors read and approved the final manuscript.

\section{Funding}

This study was supported by grants from the National Natural Science Foundation of China (81101806 and 81802367), and Shanghai Natural Science Foundation (19ZR1410200).

\section{Availability of data and materials}

Not applicable.

\section{Ethics approval and consent to participate}

This study is evaluated and approved by the Ethics Committee of Fudan University Shanghai Cancer Center (FUSCC), and written informed consent was obtained from all participants or their appropriate surrogates.

\section{Consent for publication}

Not applicable.

\section{Competing interests}

The authors declare that they have no competing interests. This manuscript is not under consideration for publication elsewhere.

\section{Author details}

${ }^{1}$ Department of Pathology, Fudan University Shanghai Cancer Center, 270 Dong An Road, Shanghai 200032, People's Republic of China. ${ }^{2}$ Department of Oncology, Shanghai Medical College, Fudan University, Shanghai, People's Republic of China. ${ }^{3}$ Institute of Pathology, Fudan University, Shanghai, People's Republic of China. ${ }^{4}$ CanHelp Genomics, Hangzhou, Zhejiang, People's Republic of China.

Received: 15 September 2019 Accepted: 11 November 2019

Published online: 21 November 2019

\section{References}

1. La Rosa S, et al. Mixed adenoneuroendocrine carcinomas (MANECs) of the gastrointestinal tract: an update. Cancers. 2012;4:11-30.

2. Lewin K. Carcinoid tumors and the mixed (composite) glandular-endocrine cell carcinomas. Am J Surg Pathol. 1987;11:71-86.

3. Mandoky L. Amphicrine tumor. Pathol Oncol Res. 1999;5:239-44.

4. Bosoteanu M, et al. Morphological and immunohistochemical characteristics of a gastric amphicrine tumor: differential diagnosis considerations. Rom J Morphol Embryol. 2011;52:485-8.

5. Reis-Filho JS, Schmitt FC. Amphicrine gastric carcinoma. Arch Pathol Lab Med. 2001;125:1513-4.

6. Jesinghaus $\mathrm{M}$, et al. Colorectal mixed adenoneuroendocrine carcinomas and neuroendocrine carcinomas are genetically closely related to colorectal adenocarcinomas. Mod Pathol. 2017;30:610-9.

7. Scardoni M, et al. Mixed adenoneuroendocrine carcinomas of the gastrointestinal tract: targeted next-generation sequencing suggests a monoclonal origin of the two components. Neuroendocrinology. 2014;100:310-6.

8. Yozu M, et al. Histologic and outcome study supports reclassifying appendiceal goblet cell carcinoids as goblet cell adenocarcinomas, and grading and staging similarly to colonic adenocarcinomas. Am J Surg Pathol. 2018;42:898-910. 
9. Brathwaite $\mathrm{S}$, et al. Mixed adeno-neuroendocrine carcinoma: an aggressive clinical entity. Ann Surg Oncol. 2016;23:2281-6.

10. Xu Q, et al. Pan-cancer transcriptome analysis reveals a gene expression signature for the identification of tumor tissue origin. Mod Pathol. 2016;29:546-56

11. Feyrter F. Über diffuse endokrine epitheliale organe. Zbl Inn Med. 1938;545:561.

12. Ratzenhofer M. Hyperplasias and tumours of the disseminated endocrine (paracrine) helle zellen Feyrter's of the gut with special regard of amphicrine cell proliferations. Verh Dtsch Ges Pathol. 1977;61:7-24.

13. Lewin KJ, Appelman HD. Endocrine cell proliferation of the stomach. Atlas Tumor Pathol. 1996;117:3-16.

14. Fenoglio-Preiser CM, et al. Carcinomas and other epithelial and neuroendocrine tumors of the large intestine gastrointestinal pathology an atlas and text. 2nd ed. Philadelphia: Lippincott-Raven Publishers; 1999. p. 909-1068.

15. Taggart MW, et al. Goblet cell carcinoid tumor, mixed goblet cell carcinoid-adenocarcinoma, and adenocarcinoma of the appendix: comparison of clinicopathologic features and prognosis. Arch Pathol Lab Med. 2015:139:782-90.

16. Wen KW, et al. Appendiceal goblet cell carcinoid: common errors in staging and clinical interpretation with a proposal for an improved terminology. Hum Pathol. 2017;65:187-93.

17. Ginori A, et al. Amphicrine carcinoma of the ampullary region. Tumori. 2015;101:e70-2.

18. Bosman FT, et al. WHO classification of tumours of the digestive system. Geneva: World Health Organization; 2010.

19. Turaga KK, Pappas SG, Gamblin T. Importance of histologic subtype in the staging of appendiceal tumors. Ann Surg Oncol. 2012;19:1379-85.

20. Edge SB, Compton C. The American Joint Committee on cancer staging manual and the future of TNM. Ann Surg Oncol. 2010;17:1471-4.

21. Gui X, et al. Goblet cell carcinoids at extra-appendiceal locations of gastrointestinal tract: an under-recognized diagnostic pitfall. J Surg Oncol. 2011;103:790-5

22. Chejfec G, et al. Amphicrine cells, dysplasias, and neoplasias. Cancer. 1985:56:2683-90.

23. Reid MD, et al. Adenocarcinoma ex-goblet cell carcinoid (appendicealtype crypt cell adenocarcinoma) is a morphologically distinct entity with highly aggressive behavior and frequent association with peritoneal/ intra-abdominal dissemination: an analysis of 77 cases. Mod Pathol. 2016;29:1243-53.

24. Tang LH, et al. Pathologic classification and clinical behavior of the spectrum of goblet cell carcinoid tumors of the appendix. Am J Surg Pathol. 2008;32:1429-43.
25. Maedler C, et al. Goblet cell carcinoid of the appendix-an interobserver variability study using two proposed classification systems. Ann Diagn Pathol. 2018:32:51-5.

26. Nugent SL, et al. Composite signet-ring cell/neuroendocrine carcinoma of the stomach with a metastatic neuroendocrine carcinoma component: a better prognosis entity. Diagn Pathol. 2007;2:43.

27. Lee LH, et al. Simplified 2-tier histologic grading system accurately predicts outcomes in goblet cell carcinoid of the appendix. Hum Pathol. 2015:46:1881-9.

28. Yang GC, Rotterdam H. Mixed (composite) glandular-endocrine cell carcinoma of the stomach. Report of a case and review of literature. Am J Surg Pathol. 1991;15:592-8.

29. Fujiyoshi $Y$, Kuhara H, Eimoto T. Composite glandular-endocrine cell carcinoma of the stomach. Report of two cases with goblet cell carcinoid component. Pathol Res Pract. 2005;200:823-9.

30. Pape UF, et al. ENETS Consensus Guidelines for the management of patients with neuroendocrine neoplasms from the jejuno-ileum and the appendix including goblet cell carcinomas. Neuroendocrinology. 2012;95:135-56.

31. Bartley AN, et al. Neuroendocrine and mucinous differentiation in signet ring cell carcinoma of the stomach: evidence for a common cell of origin in composite tumors. Hum Pathol. 2011;42:1420-9.

32. Fujiyoshi Y, Eimoto T. Chromogranin A expression correlates with tumour cell type and prognosis in signet ring cell carcinoma of the stomach. Histopathology. 2008;52:305-13.

33. Woischke $C$, et al. In-depth mutational analyses of colorectal neuroendocrine carcinomas with adenoma or adenocarcinoma components. Mod Pathol. 2017;30:95-103.

34. Jesinghaus $\mathrm{M}$, et al. Appendiceal goblet cell carcinoids and adenocarcinomas ex-goblet cell carcinoid are genetically distinct from primary colorectal-type adenocarcinoma of the appendix. Mod Pathol. 2018;31:829-39.

35. Zheng $Y$, et al. 90-gene signature assay for tissue origin diagnosis of brain metastases. J Transl Med. 2019;17:331.

\section{Publisher's Note}

Springer Nature remains neutral with regard to jurisdictional claims in published maps and institutional affiliations.
Ready to submit your research? Choose BMC and benefit from:

- fast, convenient online submission

- thorough peer review by experienced researchers in your field

- rapid publication on acceptance

- support for research data, including large and complex data types

- gold Open Access which fosters wider collaboration and increased citations

- maximum visibility for your research: over $100 \mathrm{M}$ website views per year

At $\mathrm{BMC}$, research is always in progress.

Learn more biomedcentral.com/submissions 